Illinois State University

ISU ReD: Research and eData

Theses and Dissertations

Summer 8-5-2016

\title{
Implementing a Pain Toolkit to Improve Pain Management
}

\author{
Kathleen R. Atkins DNP \\ Illinois State University, kratki2@ilstu.edu \\ Mary J. Dyck PhD \\ Illinois State University, mjdyck@ilstu.edu \\ Nancy L. Novotny PhD \\ Illinois State University, nlnov@ilstu.edu \\ Karen S. Delong MSN \\ OSF St. Joseph Medical Center, karen.s.delong@osfhealthcare.org
}

Follow this and additional works at: https://ir.library.illinoisstate.edu/etd

Part of the Nursing Commons

\section{Recommended Citation}

Atkins, Kathleen R. DNP; Dyck, Mary J. PhD; Novotny, Nancy L. PhD; and Delong, Karen S. MSN,

"Implementing a Pain Toolkit to Improve Pain Management" (2016). Theses and Dissertations. 559.

https://ir.library.illinoisstate.edu/etd/559

This Dissertation is brought to you for free and open access by ISU ReD: Research and eData. It has been accepted for inclusion in Theses and Dissertations by an authorized administrator of ISU ReD: Research and eData. For more information, please contact ISUReD@ilstu.edu. 
Implementing a Pain Toolkit to Improve Pain Management

Kathleen R. Atkins, MSN, RN, NEA-BC

Director of Clinical and Diagnostics

OSF St. Joseph Medical Center

Bloomington, IL

DNP student

Mennonite College of Nursing at Illinois State University

Normal, IL

Mary J. Dyck, PhD, RN, LNHA

Professor

Mennonite College of Nursing at Illinois State University

Nancy L. Novotny, PhD, RN, CNE Assistant Professor

Mennonite College of Nursing at Illinois State University

Karen DeLong, MSN, RN, CPN, CNL

Performance Improvement Facilitator and 6 Sigma Black Belt

OSF St. Joseph Medical Center

Bloomington, IL 


\section{Abstract}

OBJECTIVES: The purpose of this study was to evaluate the effect of a pain toolkit to improve pain management.

BACKGROUND: The World Health Organization and Joint Commission state effective pain management is a basic human right.

METHODS: Patients' ratings of pain management on a 25-bed orthopedic/neurology unit three months before and after implementation of a toolkit were compared. The toolkit included bedside report, care board, purposeful hourly rounding, pain algorithm, pain status indicator, and complex pain interventions. The data are comprised of patient responses to the following Hospital Consumer Assessment of Healthcare Providers and Systems survey questions: pain control and staff's help with pain.

RESULTS: There was statistically significant improvement in patients' perceptions that staff "always" did everything to help with pain. There was no statistically significant improvement in patients' perceptions that pain as "always" well controlled.

CONCULSIONS: The toolkit is a valuable resource in improving pain management. 


\section{Problem Statement}

During the last three months of 2015 on the Orthopedic/Neurology unit in a 149 bed Magnet designated medical center $74 \%$ of respondents indicated "always" to "staff does everything to help with pain" and $50 \%$ responded "always" to "pain controlled" on Hospital Consumer Assessment of Healthcare Providers and Systems (HCAHPS) surveys". A gap analysis showed evidence based practices were not routinely used on this unit. There existed an opportunity to implement bedside report, care boards with pain management information, pain algorithm, electronic medical record (EMR) to identify patient's pain status, purposeful hourly rounding, and complex pain strategies using patient engagement and shared decision making.

To address the problem, this project was implemented using a pain toolkit with evidence based practices. The objective was to teach the staff how consistent use of evidence based tools could impact patients' pain management with a patient outcome of improved pain management.

\section{Literature Review}

Several studies have shown that patients' pain treatment is inadequate due to uncertain diagnosis and lack of clinician's knowledge related to best practices to treat pain (1-3). Barriers to effective pain management for hospitalized patients include poor communication among health team members, poor communication between patients and the health care team, lack of organizational commitment to pain management, limited reimbursement for interdisciplinary care and lack of knowledge related to pain management $(4,5)$.

Patient outcomes show improvement when nurses' practice in an evidence-based manner. The best pain management practices are patient-centered with nurses utilizing good communication skills and shared decision-making with patients (6). Studies linked several practices with improved pain management. These included providing patients with information on options for pain relief, informing patients when to expect the next dose, improving nurses' knowledge of analgesia (4, 6-9) establishing an interdisciplinary pain team (10), hourly rounding (10) and instituting daily patient pain rounding (6). Nursing communication techniques can also improve patient engagement in management of their pain $(9,10)$. These techniques include bedside report, care boards, purposeful hourly rounding (5); documentation of methods and frequency of pain assessments, utilization of specific guides for pain medications, 
maintaining accountability for pain management through use of pain algorithms and promising patients to keep their pain under control $(4,6,9,10)$.

\section{Framework}

The lowa Model of Evidence Based Practice (11) was utilized for the implementation of a pain toolkit to improve pain management. This model facilitates the application of empirical evidence to nursing practice so knowledge focused problems can be evaluated from a research perspective.

\section{Methods}

\section{Design and Sample}

A quasi-experimental, pre/post design was used to compare pain management of patients on a 25-bed Orthopedic/Neurology unit in a community medical center pre and post pain toolkit implementation. Institutional Review Boards at the University of Illinois College of Medicine at Peoria and Illinois State University approved the project. The samples included adults, 18 years of age or older, who were discharged to home after being inpatients on the Orthopedic/Neurology unit. The pre-implementation sample included those captured between October through December, 2015. The post-implementation sample included those captured between February through April, 2016.

\section{Intervention}

A pain toolkit was developed by a team of nursing leadership and performance improvement members who were convened to address the lack of pain management reported by the HCAHPS data. First the team performed a literature search and then evidence was appraised for the best pain management practices. An interdisciplinary team including bedside nurses, a care management nurse, nurse leaders, a nurse educator, pharmacist, and a physical therapist participated in the planning, implementation and learning activities.

First, an electronic learning module (ELM) was developed and for completion by the Orthopedic/Neurology and Float registered nurses. The ELM incorporated education about acute and chronic pain, expected actions and behaviors for all staff related to pain management and the six evidence based practices in the pain toolkit. These evidence-based practices included bedside report, care boards with pain management details, purposeful hourly rounding, EMR with patient's pain status, pain algorithm, and complex pain control strategies. 
Second, the team invited one novice nurse and one nurse with twenty years of experience to participate in a trial run of a fair which would be used to expose nurses in the involved units to this toolkit. The two nurses were asked to provide feedback about their participation and discuss how using the pain toolkit would improve pain management. Five fair stations were set up to acquaint the nurses with the need for a change and educate them about each tool. Time was planned to allow participants to perform a return demonstration of their use of the tools. Based on feedback provided during this trial run, the team revised fair plans to include more group discussion at the bedside report and care board stations.

Third, the pain toolkit fair was held to train all nurses in the project over a two-week period during January 2016. Discussions at each pain toolkit station were limited to eight participating nurses. At the first station, the problem was introduced by discussing the current unit HCAHPS pain data. At the second station the pain algorithm was introduced using scenarios of patients with uncontrolled pain. The pain algorithm highlighted use of alternative non-pharmacological interventions and the nurses were encouraged to place a pharmacy consult as part of the team approach to pain control. At the third station each nurse set up the EMR pain status indictor on a computer. A team member moderated discussion on the poster illustrating complex pain control strategies was located at the fourth station. At the last station each nurse was given a patient scenario and then required to practice bedside report using patient engagement and shared decision making techniques together with the new care boards. Documentation on the new care boards included the patient's pain goal, pain score, name and dose of pain medication and time for next dose. Discussions with the nurses included how to respond when patients say they want to have zero pain, what to write on the care board, and how to discuss unrealistic pain goals with patients. This final station also required the nurse to practice using key words about pain with purposeful hourly rounding as they related to the patient scenarios.

After training, the team observed staff using pain toolkit practices three times over two months. The data collected during observations comprised: using the pain algorithm; documenting on the care board of the pain goal, the last dose of pain medication, and the time of the next dose; setting up the EMR pain status indicator; and discussing pain during bedside report. Following the observations staff was recognized by the implementation leader for using the pain toolkit tools and coached how to improve their use of the tools. 


\section{Measurement and Data Collection}

The HCAHPS survey was used to measure the outcome of pain management. HCAHPS is the national standard for collecting, reporting, and benchmarking information about hospitalized patient's experiences (12). The National Quality Forum has endorsed the HCAHPS survey (13). The HCAHPS survey has 27 Likert-type questions asking the patients to evaluate the frequency of certain behaviors during their inpatient stay. In terms of pain management, patients are asked 1) how well pain was controlled and 2) if staff did everything possible to help with pain. Response choices were "always, usually, sometimes, or never" for the two questions. Press Ganey ${ }^{\circledR}$ is the hospital vendor and receives a nightly file which identifies inpatients who were greater than 18 years of age, and discharged to home. Within three business days, Press Ganey® randomly selects $30 \%$ of the qualified patients to be mailed a survey with a self-addressed stamped return envelope to Press Ganey®. The investigator downloaded Excel reports from the Press Ganey ${ }^{\circledR}$ Improvement Portal. The reports provided a summary of the number and frequency of patient responses to each item during the pre and post implementation periods.

\section{Data analysis}

The implementation team met and reviewed the findings from the observations. Themes identified were inconsistent use of care boards, lack of practice using pain algorithm, inconsistency with including patients with bedside report. The implementation team focused performing direct observation of bedside report and using recognition cards for use of care boards showing pain information and use of pain algorithm. The focus recognition cards acknowledged staff for using the pain toolkit practices. HCAHPS Excel file data were imported into SPSS 22.0. For both questions, a small number of responses were identified for several response options. For this reason, dichotomous variables were created by retaining the always category and collapsing the response options of usually, sometimes, and never into not always. Pearson Chi-square was used to compare rates of patients who responded always to each item before and after implementation of the pain toolkit. The level of significance was set at $p<.05$.

\section{Results}

The team performed validation of the nurses using the toolkit. The validation occurred by having discussions with the nurses on how they were doing using the tools. The validation changed to direct observations of the nurses performing bedside report, including the patient in the bedside report and 
using of the care boards. Validation on use of pain algorithm, use of EMR pain status indicator and hourly rounding were by discussion. The nurses voiced it was new behavior with bedside report to include the patient. Nurses wrote on the care boards, however, they needed coaching to explain the care board to the patient after recording the pain medication and time for next dose. Nurses agreed with the need to practice evidence based communication, but found that including the patient was a new skill. Nurses voiced concern that writing the time for the next pain medication on the board might cause anxiety for the patient if the nurse was late in bringing the medication to the bedside. The pain algorithm was practiced using scenarios and led to discussion about opioid use.

Early observations of nursing staff showed EMR pain status indicator was set up to show pain status. Observations with bedside report showed inconsistencies with patient inclusion in the discussion with the oncoming nurse about what worked well during the shift to control their pain. There were gaps on the care board completing pain medication, pain goal, pain score and time for next pain medication. Additional observations included lack of hourly rounding on pain and lack of pain algorithm use, including implementing a pharmacy consult for uncontrolled pain.

A second round of observations showed improvement with the nurses using the pain toolkit. Comments reported by observers were more recognition cards for using the care board for keeping patients informed and asking patients at bedside report how pain is being controlled. An example of a recognition card from observer to nurse "Allison had a patient on multiple pain medications. She did an excellent job keeping the care board updated with pain management details and using it to communicate to the patient. Her commitment to controlling her patient's pain is very apparent." In a third round of nursing staff observations, nursing staff showed patients' comfort goal on the EMR, pain issues shared with providers, pain goals and time for next pain medication on care boards, and patient engagement during bedside report. Purposefully hourly rounding, using pain algorithm and complex pain control strategies were inconsistently utilized as part of the pain toolkit.

A total of 54 patients returned surveys from October through December and 43 were returned from February through April 2016. There was a statistically significant improvement in patients' perceptions that staff always did everything to help with pain (Chi square $=4.377, \mathrm{df}=1, \mathrm{p}=.04$, with an increase from $74.1 \%$ before implementation to $90.7 \%$ after implementation of the toolkit. Patients' who perceived pain 
was always controlled increased from $50 \%$ before implementation to $67.4 \%$ afterwards. This change, however, was not statistically different (Chi square $=2.98, d f=1, p=.08)$. See Figure 1 .

\section{Discussion}

Use of the pain toolkit improved patients' perception of pain management. These findings are congruent with the findings of similar studies by several authors. For example, Saver (9) found setting expectations for managing patients' pain control, setting realistic expectations, establishing pain goals, using bedside handoffs that includes the patient, using a pain algorithm that the odds of a patient being satisfied were 4.86 times greater if pain was controlled and 9.92 times greater if staff performance was appropriate. This means how staff reacted was even more important than if the pain was actually controlled. Aston (14) reported findings from a Johns Hopkins study which examined the relationship between patients' perception of pain controlled during their hospitalization and overall satisfaction. The findings were patients rated their overall experience higher if they perceived their care providers did everything to control pain rather than the pain actually being controlled. This study supports the previous work by Ketelsen and colleagues (4) who described best pain management practices of patient engagement and involving the patient's with setting pain goals with the evidence based practices of bedside report care boards, and purposeful hourly rounding. The results of this project were consistent with the literature regarding benefits of using bedside report and including the patient, establishing pain goals with the patient, setting realistic expectations and goals, using a pain algorithm (9). The pain toolkit implemented evidence based practices using the lowa Model of Evidence-Based practice framework which reinforced IOM pain care initiatives and The Joint Commission mandated pain standards practices. Consistent results were also found by (15).

Several studies have tested pain management practices, and patient satisfaction levels. Idell, Grant and Kirk's (16) study to improve nursing pain reassessment used a research utilization model and advance practice nurses. They found allowing staff to create unit specific solutions, use of research in bedside care, aligning pain reassessment practices with clinical pain guidelines promoted improved pain management through better reassessment documentation. Chung \& Lui (17) conducted a prospective survey in a 1200 bed hospital on adult patients admitted to Hong Kong for surgery and found the best way to understand if pain management is adequate to ask the patient and this view is supported by the 
Agency for Health Care Policy and Research (AHCPR). They used a patient outcome questionnaire developed by the American Pain Society to gather data about patients' pain and satisfaction with pain relief. Approximately $85 \%$ complained about pain during 24 hour prior to pain assessment of pain, $80 \%$ indicated both the nurses and physician reminded them to report pain when it occurred and $48.6 \%$ agreed that nurses and physician emphasized the importance of pain relief. $65 \%$ of subjects were satisfied with level of health care providers, regarding their postoperative pain management.

This evidence based project showed using evidenced base communication techniques while using evidence based practices showed a statistically significant improvement in patients' perceptions staff's help with pain. Patients, who perceived pain control increased, did report more "always" to pain control after pain toolkit implementation which can be argued clinical significant however not statistically significant.

\section{Limitations}

All of the evidenced based practices introduced in the pain toolkit were new practices for the nurses. Nurses on the unit did not previously include patients in conducting bedside report, include pain information on care boards, or record pain status indicators in the EMR. Educating multiple new practices all at once and in a single educational session may have been burdensome to the nurses and not have allowed them sufficient time to become proficient with the all tools. In addition, the team members who performed observations had limited training on coaching staff using the pain toolkit.

This project did not include examination of the reliability and validity of the HCAHPS items. The small sample sizes may have limited the ability to identify a statistical difference even if one existed. Finally, the pre/post samples may not have had similar pain to manage.

\section{Implications for Nurse Leaders}

Nurse leaders recognize the challenges faced by nurses as they assist patients in managing their pain. Providing feedback and rationales for the use of evidence based tools supports nurses as they manage their patients' pain. The nurse leader's role includes monitoring use of pain toolkit tools for effectiveness, recognizing the staff for employing evidenced base tools, and providing feedback to improve consistent use of the tools for sustained pain management.

\section{Conclusion}


The HCAHPS questions, "how well pain controlled" and "staff did everything to help with pain", indicate pain management is more than giving pain medications. Overall, the use of the pain toolkit improved pain management as measured by HCAHPS surveys. More patients responded "always" after pain toolkit implementation to the pain questions. This supports the idea that use of evidence based practices of bedside report, care board with pain management information, purposefully hourly rounding on pain, EMR with pain indicator, pain algorithm, and complex pain strategies can positively impact the patient's perception of pain management. Using the pain toolkit containing evidence based practices can improve the patient's perception of pain management during their hospital stay. 
References

1. Goldberg, DS, McGee, SJ. Pain as a global public health priority. BMC Public Health. 2011, 11.

2. Gupta, A., et al. Patient perception of pain care in the United States: A 5-year comparative analysis of hospital consumer assessment of health care providers and systems. Pain Physician. 2014, 17(5):369-377.

3. Institute of Medicine (IOM). Relieving pain in America: A blueprint for transforming prevention, care, education, and research. Washington, D.C.: The National Academies Press; 2011.

4. Ketelsen, L, Cook, K, Kennedy, B. The HCAHPS handbook 2: Tactics to improve quality and the patient experience. Fire Starter Publishing; 2014.

5. Dempsey, C, Reilly, B, Buhlman, N. Improving the patient experience: Real-world strategies for engaging nurses. J Nurs Adm. 2014, 44(1):142-151.

6. Glowacki, D. Effective pain management and improvements in patients' outcomes and satisfaction. Critical Care Nurse. 2015, 35(3).

7. Hayes, K., Gordon, DB. Delivering quality pain management: The challenge for nurses. AORN J, 2015, 101(3):327-337.

8. Mallick-Searle, T, et al. Peering through the mask of pain. Stanford Nurse. 2012, 32(1):4-6.

9. Saver, C. Pain and patient experience: A business partnership. OR Manager. 2013, 29(6):1.

10. Long, L. Impressing patients while improving HCAHPS, Nurs Manag, 2012, 43(12):32-37.

11. Taylor-Piliae, R. Utilization of the lowa model in establishing evidence-based nursing practice. Intensive and Critical Care Nurs. 1999, 15(6):357-362.

12. Giordano, L.A., et al. Development, implementation, and public reporting of the HCAHPS survey. Med Care Res Rev. 2010, 67(1):27.

13. NQF endorses HCAHPS patient perception survey. Healthcare Benchmarks Qual Improve. 2005, 12(7):82-83.

14. Aston, G. Smart pain management makes good business sense. H\&HN: Hosp Health Networks. 2012, 86(6):38-50.

15. Ramira, ML, Instone, S, Clark, MJ. Pediatric pain management: An evidence-based approach. Qual Improv. 2016, 42(1):39-46. 
16. Idell, CS, Grant, M, Kirk, C. Alignment of pain reassessment practices and national comprehensive care network guidelines. Oncology Nurs For. 2007, 34(3):661-671.

17. Chung, JW, Lui, JC. Postoperative pain management: Study of patients' levvel of pain and satisfaction with healthcare providers' responsiveness to their report of pain. Nurs Health Sci. 2003. 5:13-21. 